\title{
The sequential management of recurrent temporomandibular joint ankylosis in a growing child: a case report
}

\author{
Jung-Won Cho, Jung-Hyun Park, Jin-Woo Kim and Sun-Jong Kim*
}

\begin{abstract}
Background: Temporomandibular joint (TMJ) ankylosis in children often leads to facial deformity, functional deficit, and negative influence of the psychosocial development, which worsens with growth. The treatment of TMJ ankylosis in the pediatric patient is much more challenging than in adults because of a high incidence of recurrence and unfavorable growth of the mandible.

Case report: This is a case report describing sequential management of the left TMJ ankylosis resulted from trauma in early childhood. The multiple surgeries including a costochondral graft and gap arthroplasty using interpositional silicone block were performed, but re-ankylosis of the TMJ occurred after surgery. Alloplastic TMJ prosthesis was conducted to prevent another ankylosis, and signs or symptoms of re-ankylosis were not found. Additional reconstruction surgery was performed to compensate mandibular growth after confirming growth completion. During the first 3 years of long-term follow-up, satisfactory functional and esthetic results were observed.

Conclusions: This is to review the sequential management for the recurrent TMJ ankylosis in a growing child. Even though proper healing was expected after reconstruction of the left TMJ with costal cartilage graft, additional surgical interventions, including interpositional arthroplasty, were performed due to re-ankylosis of the affected site. In this case, alloplastic prosthesis could be an option to prevent TMJ re-ankylosis for growing pediatric patients with TMJ ankylosis in the beginning.
\end{abstract}

Keywords: TMJ ankylosis, Recurrent ankylosis, Pediatric patient TMJ ankylosis

\section{Background}

Temporomandibular joint (TMJ) ankylosis can be defined as the union of mandibular condyle to the cranial base which is the articular surface through osseous or fibrous tissue, with partial or complete mandibular impediment [1]. The etiological factors for TMJ ankylosis include trauma, rheumatoid arthritis, congenital anomalies, infection, and neoplastic processes. Trauma is well known as the most predominant factor in TMJ ankylosis particularly in children and is associated with inadvertent use of forceps during delivery, traffic accident, and falls [2-4]. When TMJ ankylosis occurs in children, the future growth and development of the jaws and teeth are affected

\footnotetext{
*Correspondence: oralsurgeonsj@gmail.com

Department of Oral and Maxillofacial Surgery, Ewha Womans University Medical Center, 1071, Anyangcheon-ro, Yangchen-gu, Seoul 07985, South Korea
}

negatively. Furthermore, psychosocial development of the children affected is profoundly influenced due to the obvious facial deformity, which worsens as they grow [5]. Condyle reconstruction is carried out in order to restore TMJ function and facial deformity in adults, whereas high incidence of recurrence and the probable change in the unfavorable growth of the mandible are also needed to be considered in children $[5,6]$.

It is generally recommended that as soon as the condition is diagnosed, the surgery of TMJ ankylosis should be initiated. The main purpose of the surgery is the reestablishment of joint and harmonious jaw functions in children [5, 7]. This case report presents sequential management of recurrent TMJ ankylosis with a variety of methods in a growing child.
Springer Open

(C) 2016 The Author(s). Open Access This article is distributed under the terms of the Creative Commons Attribution 4.0 International License (http://creativecommons.org/licenses/by/4.0/), which permits unrestricted use, distribution, and reproduction in any medium, provided you give appropriate credit to the original author(s) and the source, provide a link to the Creative Commons license, and indicate if changes were made. 


\section{Case presentation}

A 12-year-old male patient was referred to the Department of Oral and Maxillofacial Surgery at Ewha Womans University Mokdong Hospital for evaluation and treatment of left TMJ ankylosis. He had been diagnosed with bony ankylosis of the left TMJ due to trauma at the age of 1. At 8 years of age, the patient had received TMJ gap arthroplasty with condylectomy at a different hospital. However, he had a difficulty in opening his mouth since ankylosis of the left TMJ recurred. On the initial clinical examination, maximum mouth opening (MMO; maximum interincisal distance) was less than $2 \mathrm{~mm}$ (Fig. 1). The panoramic radiograph revealed bone mass of the left mandibular condylar process (Fig. 2a). There were irregular expanded bony contour, cortical thickening, and diffused sclerosis around the left TMJ on threedimensional computed tomography images (Fig. 2b). Increased uptake in the left TMJ were observed in bone scan (Fig. 2c). These diagnostic images confirmed a true bony ankylosis of the left TMJ, which was assessed as type IV TMJ ankylosis according to Sawhney's classification [8].

\section{First procedure, 12 years old; costochondral graft}

Removal of ankylotic mass and TMJ reconstruction with costochondral graft were planned. The left TMJ was approached through a preauricular incision, and excision of ankylotic mass was done. The costochondral graft, harvested from the fifth rib of the right side, was adjusted to the condyle area. It was carefully done so the

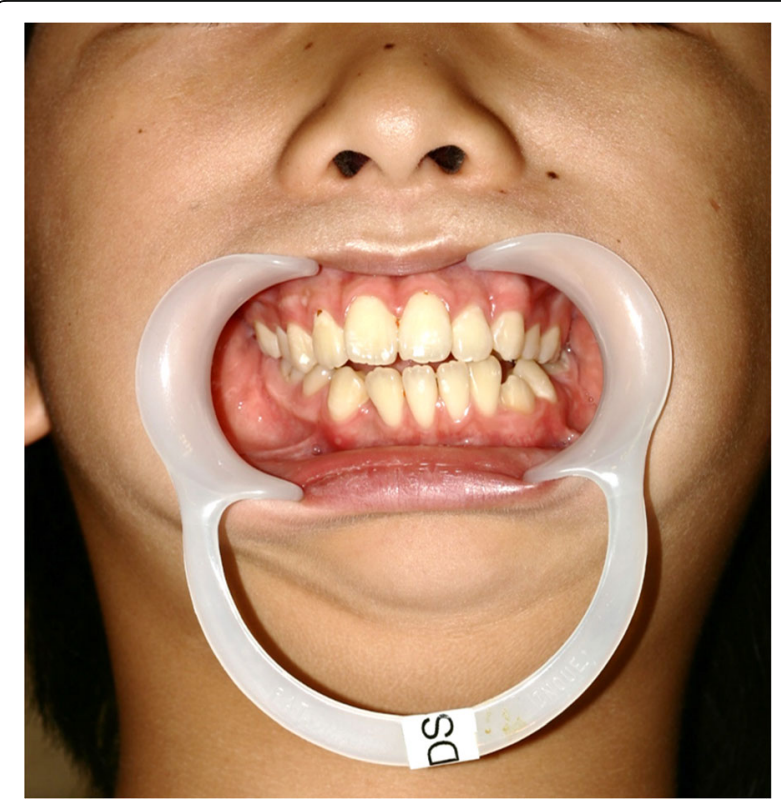

Fig. 1 Frontal view after the formation of re-ankylosis when he was 12 years old cartilaginous part of the graft is not separated from the bone. A temporalis muscle fascia flap was rotated over the arch into the joint and was lined in the TMJ space in order to reconstruct the roof of the new glenoid fossa. The deep temporalis fascia and the superficial muscle layer were transferred to construct a barrier, to support the function of the reconstructed ramus/condyle unit and to maintain flap vascularity. Five bicortical screws were secured on the left mandibular ramus for rigid internal fixation of grafted bone (Fig. 3).

A postoperative MMO measured up to $20 \mathrm{~mm}$, and it was increased to $26 \mathrm{~mm}$ after 5 weeks postoperatively. However, newly growing bone encapsulated the grafted costochondral head resulting in a limited mouth opening of less than $12 \mathrm{~mm}$ within a year postoperatively. Radiographic and clinical evidences confirmed re-ankylosis of the left TMJ. Computed tomography revealed bony ankylosis on the left TMJ (Fig. 4). Increased uptake lesion in the mid-ascending ramus area of the left mandible was also observed in the bone scan.

\section{Second procedure, 13 years old; gap arthroplasty with interpositional silicone block}

Removal of costochondral graft and gap arthroplasty using interpositional silicone block was carried out when the patient was 13 years of age. A 12-mm-thick silicone block with full coverage of the glenoid fossa was placed and fixed in the cranium (Fig. 5). A postoperative MMO measured up to $26 \mathrm{~mm}$ in the 3 weeks postoperative follow-up. However, MMO was decreased to $18 \mathrm{~mm}$ after 1 year postoperatively. There was also gradual reduction of mouth opening, and MMO was noted to be $12 \mathrm{~mm}$ after 1 year and 4 months postoperatively. Radiographic finding also revealed the re-ankylosis of the left TMJ. Therefore, alloplastic temporomandibular joint reconstruction combined with partial mandibulectomy was planned.

\section{Third procedure, 15 years old; reconstruction with alloplastic condyle}

Partial mandibulectomy and placement of metallic condylar head prostheses were performed (Fig. 6). After 1 year postoperative follow-up, bone scan revealed the absence of abnormal bone uptake in the reconstructed TMJ area. Mouth opening was consistently measured up to $40 \mathrm{~mm}$ without any signs or symptoms of re-ankylosis.

\section{Fourth procedure, 17 years old; reconstruction of mandibular ramus with iliac bone}

Reconstruction of the mandibular ramus with corticocancellous iliac bone graft was performed to compensate additional growth of the mandible after confirming the facial growth completion through a serial cephalometric 

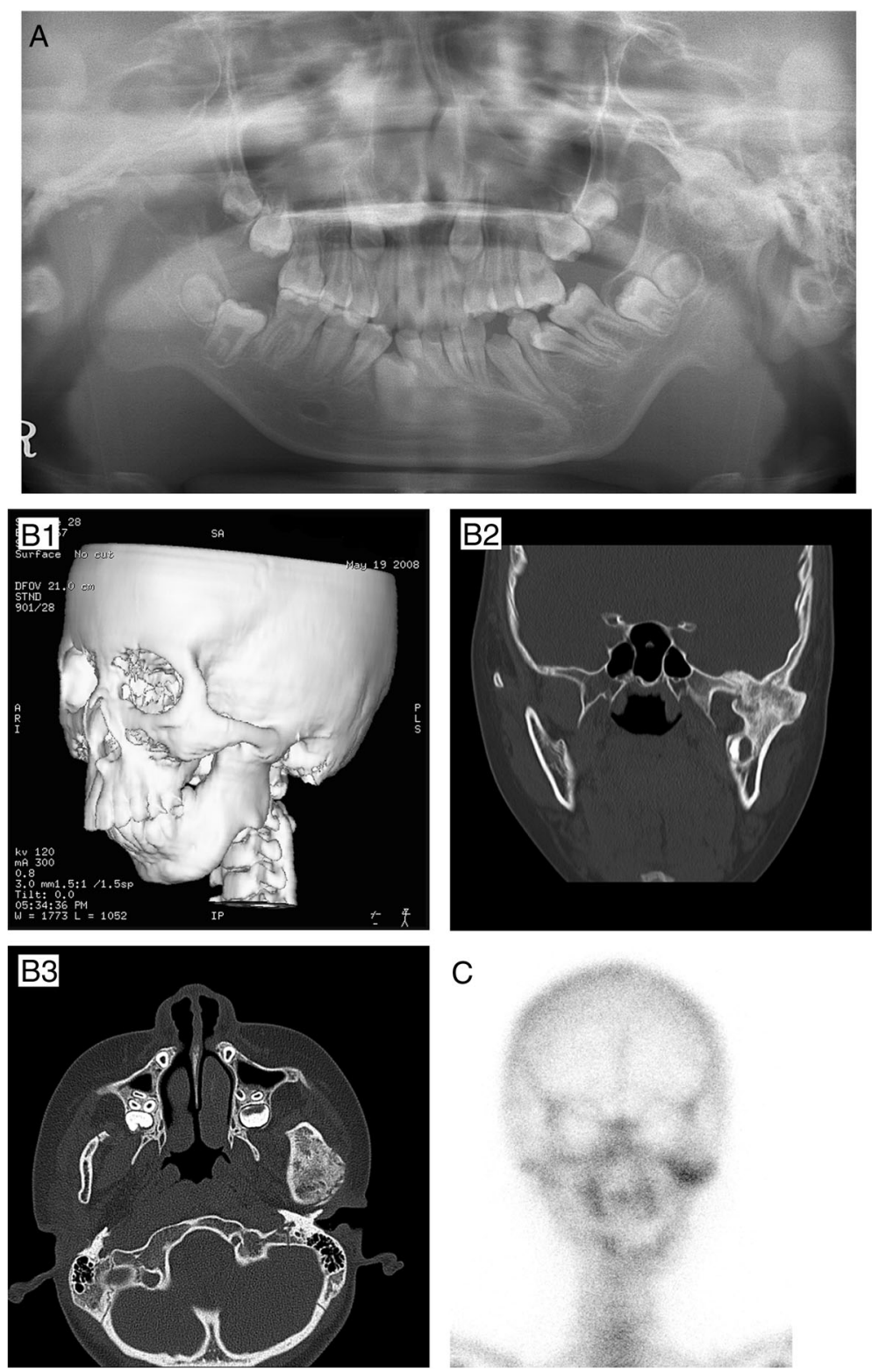

Fig. 2 Initial radiographic evaluation. a Preoperative panoramic radiograph. Bone mass of the left mandibular condylar process. b Preoperative three-dimensional computed tomography. Irregular expanded bony contour, cortical thickening, and diffused sclerosis around the left TMJ. c Preoperative three-dimensional computed tomography. Irregular expanded bony contour, cortical thickening, and diffused sclerosis around the left TMJ

analysis at 17 years of age (Fig. 7). During clinical examination at 6 months postoperatively, the patient showed a good range of motion with MMO of $35 \mathrm{~mm}$. The patient had a long-term follow-up of orthodontic treatment for occlusal stabilization consistently, and MMO was being maintained at $35 \mathrm{~mm}$ during the first 3 years of follow-up (Fig. 8a). Mandibular asymmetry (Fig. 8b) and the evidence of re-ankylosis on radiographic evaluation (Fig. 8c) were not observed.

\section{Discussion}

This case report introduces sequential management of the left TMJ ankylosis resulted from trauma in early childhood. TMJ reconstruction was carried out using costal cartilage graft after removing ankylosed tissues of the left TMJ. The use of costochondral graft is a common practice for condyle reconstruction in children with ankylosis. The advantages of this procedure include biologic and anatomic similarity to the mandibular 


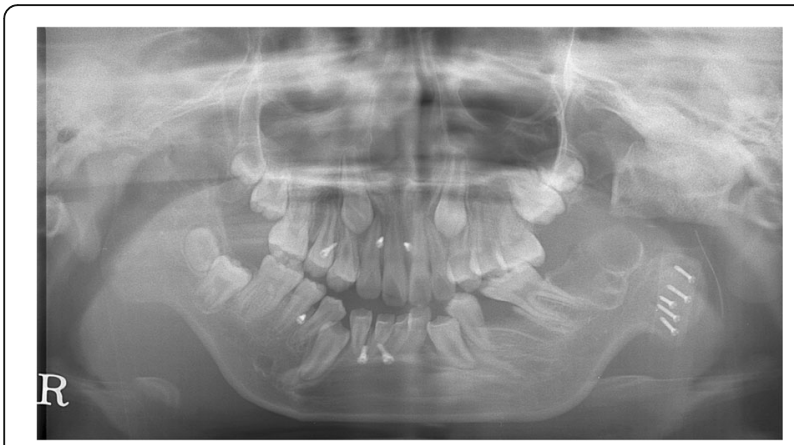

Fig. 3 Postoperative panoramic radiograph after surgery. The costochondral graft was adjusted to the left condyle area and secured on the left mandibular ramus by five bicortical screws for rigid internal fixation

condyle, growth potential in pediatric patients, ease of harvesting and adapting the graft, and low morbidity of the donor site $[7,9]$. Because of the similarities of its primary and secondary cartilages to those of the mandibular condyle [9], the costochondral graft will provide growth potential and keep pace with the growth of the unaffected side, maintaining mandibular symmetry throughout growth [7]. However, long-term studies on mandibular growth in children with reconstructed TMJs using costochondral grafts show excessive growth on the treated side, occurring in $54 \%$ of the 72 cases evaluated, and only $38 \%$ of the cases presented equal growth with the opposite side, and ankylosis can be expected in rare instances from the recipient site [10-12].

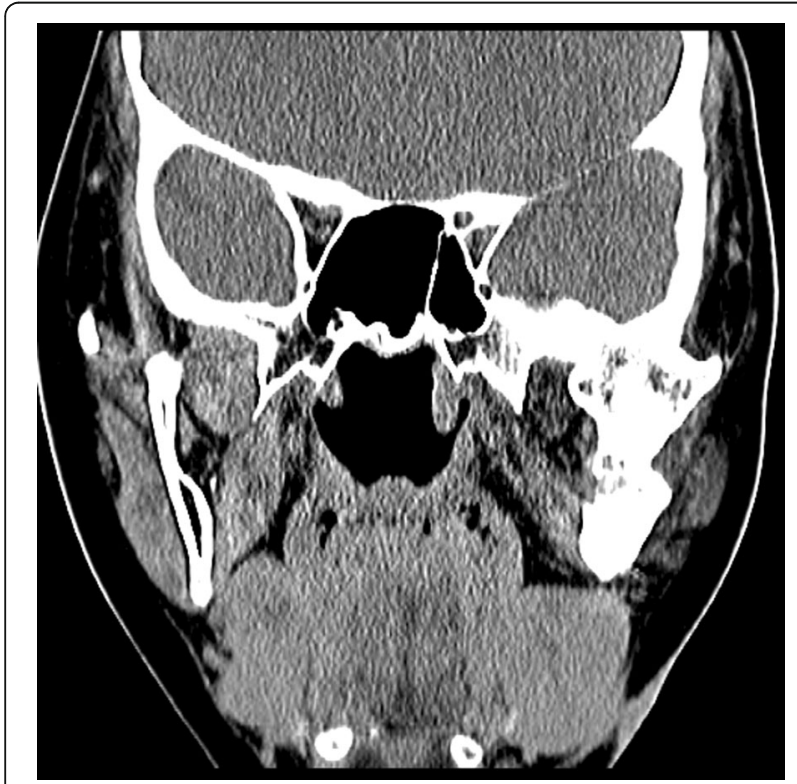

Fig. 4 Computed tomography after 1 year postoperatively. Bony ankylosis was confirmed on the left TMJ

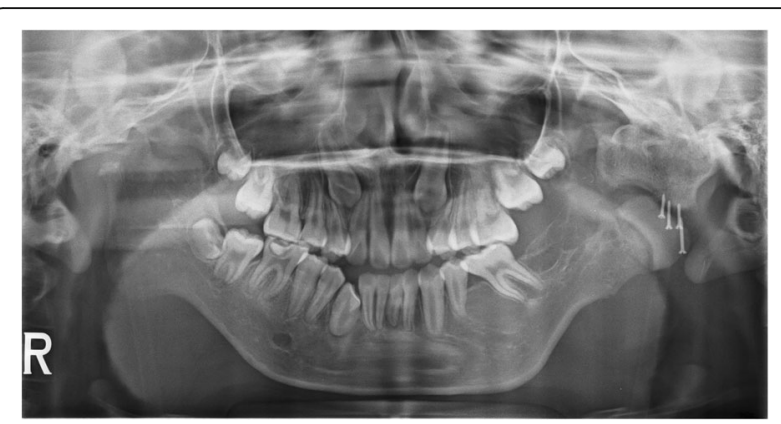

Fig. 5 Postoperative panoramic radiograph after surgery. Silicone block with full coverage of the glenoid fossa was placed and fixed in the cranium

It is recommended that early mobilization and aggressive physiotherapy should be done after releasing the intermaxillary fixation (IMF) and immediately postoperatively for patients reconstructed with the costochondral graft [5]. In this case, there were radiographic and clinical evidences confirming re-ankylosis on the recipient site after 1 year postoperatively and mainly due to the IMF with elastic over 8 weeks after surgery and noncompliance with proper physiotherapy.

Even though proper healing was expected after reconstruction of the left TMJ with costal cartilage graft, additional surgical interventions, including interpositional arthroplasty, were performed due to re-ankylosis of the affected site. There is no consensus in the literature on a standard protocol for management of TMJ ankylosis, but three modalities are commonly used: (1) gap arthroplasty, (2) interpositional arthroplasty, and (3) excision and articular reconstruction [13]. The first modality is performed without intervening grafts or materials and is based on resection of ankylosed bone. According to the literature, a minimum of $15-\mathrm{mm}$ gap is recommended between the recontoured glenoid fossa and the mandible for preventing re-ankylosis [14, 15]. Gap arthroplasty offers an advantage of a simple procedure

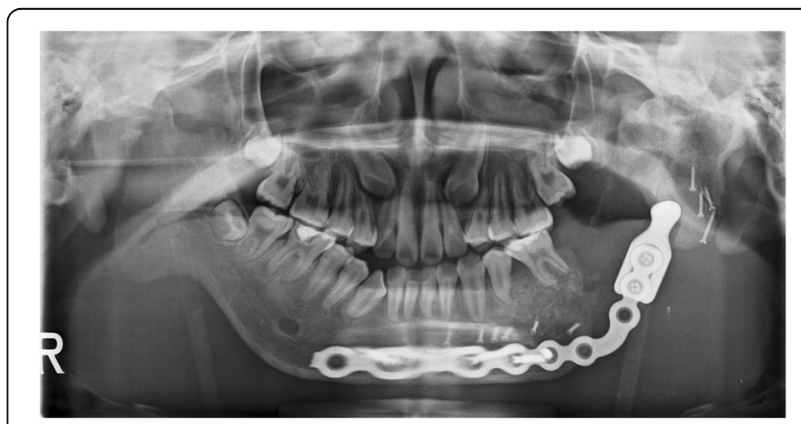

Fig. 6 Postoperative panoramic radiograph after surgery. Alloplastic temporomandibular joint reconstruction combined with partial mandibulectomy was performed 


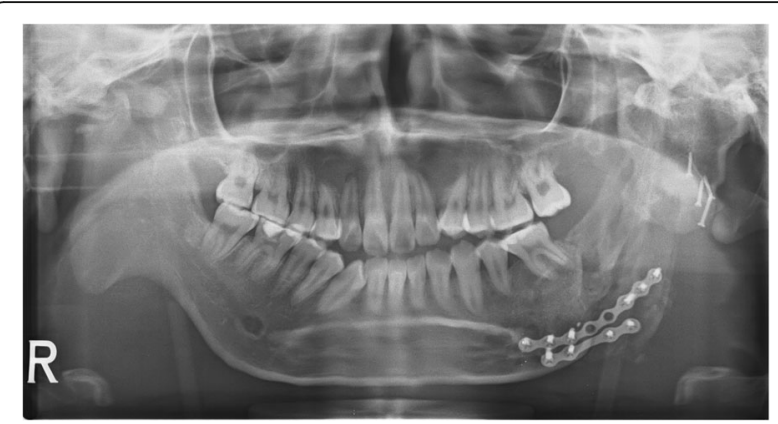

Fig. 7 Postoperative panoramic radiograph after surgery. Reconstruction of the mandibular ramus with cortico-cancellous iliac bone graft was performed

and requires a short surgical time. However, disadvantages include the following: (1) creation of a pseudoarticulation, (2) a short mandibular ramus with anterior open bite in bilateral cases and posterior open bite in unilateral cases, (3) failure of removal of pathologic bone tissue, and (4) high risk of recurrence [16, 17]. The interpositional arthroplasty is recommended after gap arthroplasty as a means to limit resection and recurrence. In this procedure, autogenous and alloplastic materials are placed in the osteotomized area. The important criteria in the choice of graft or interpositional material are cost, esthetic consequences after graft removal, long-term behavior, risk of infection, biocompatibility, tolerance, and prevention of recurrence [16]. In a comparative study, satisfactory results were observed in $92 \%$ of cases with skin graft [18] and $83 \%$ of cases with temporal muscle flaps [13]. Among the several alloplastic materials, gold foil, silastic sheet, acrylic, stainless steel, and silicone prostheses have been used [19-21].

Alloplastic temporomandibular joint replacement can provide a viable option for the multiple operated patients with distorted TMJ anatomy or severe anatomical discrepancies involving the TMJ with recurrent ankylosis $[22,23]$. Orthopedic surgeons often prefer alloplastic prosthesis in the replacement of joint in similar situations involving other joints over the use of autogenous bone into the area where reactive or heterotropic bone is forming [24]. In this case, alloplastic prosthesis could be a good selection to prevent recurrent TMJ ankylosis in a growing child.

\section{Conclusion}

It is proposed that alloplastic prosthesis could be performed to prevent TMJ re-ankylosis for growing pediatric patients with TMJ ankylosis in the beginning. And then there is an additional surgery to compensate mandibular growth after confirming growth completion.
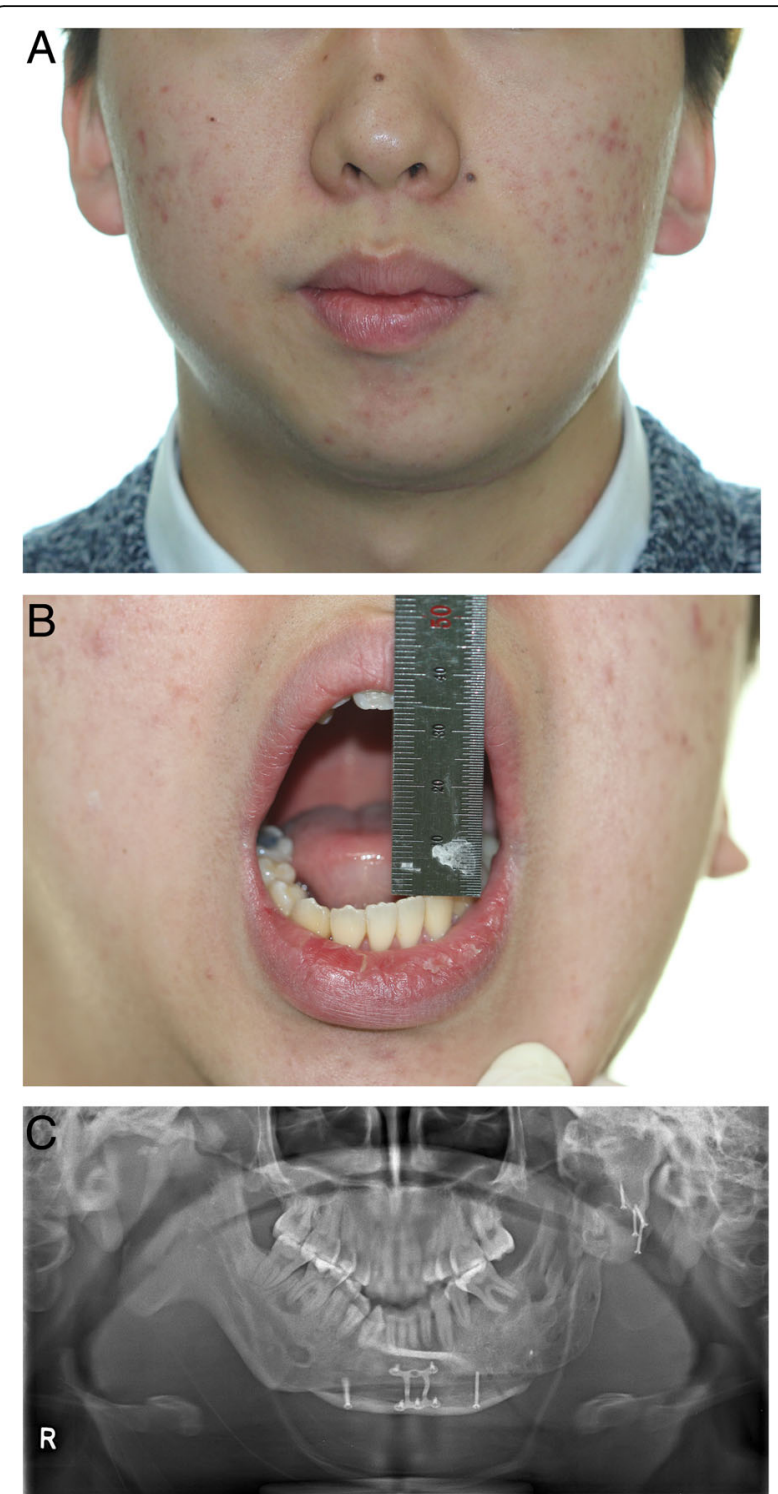

Fig. 8 The 3 years of long-term follow-up after last surgery. a Frontal view when he was 19 years old. Mandibular asymmetry was not observed. b Maximum mouth opening was noted to be $35 \mathrm{~mm}$ and $\mathbf{c}$ the evidence of reankylosis was not observed on panoramic radiograph after 3 years postoperatively

\section{Acknowledgements}

This study received no specific grant from any funding agency in the public, commercial, or not-for-profit sectors.

\section{Authors' contributions}

J-WC, J-HP, and J-WK are responsible for the data collection, drafting the article, and the critical revision of the article. S-JK is responsible for the conception and design of the study, the critical revision of the article, and the approval of the article. All authors read and approved the final manuscript.

Competing interests

The authors declare that they have no competing interests. 


\section{Consent for publication}

Written informed consent was obtained from the patient for the publication of this report and any accompanying images.

\section{Ethics approval and consent to participate} Not applicable.

Received: 21 June 2016 Accepted: 18 September 2016

Published online: 05 October 2016

\section{References}

1. de Andrade LHR, Cavalcante MAA, Raymundo R, de Souza IPR (2009) Temporomandibular joint ankylosis in children. J Dent Child 76:41-45

2. Balaji S (2003) Modified temporalis anchorage in craniomandibular reankylosis. Int J Oral Maxillofac Surg 32:480-485

3. Güven O (1992) Fractures of the maxillofacial region in children. J CranioMaxillofac Surg 20:244-247

4. Oztan HY, Ulusal BG, Aytemiz C (2004) The role of trauma on temporomandibular joint ankylosis and mandibular growth retardation: an experimental study. J Craniofac Surg 15:274-282

5. Kaban LB, Bouchard C, Troulis MJ (2009) A protocol for management of temporomandibular joint ankylosis in children. J Oral Maxillofac Surg 67: 1966-1978

6. Güven O (2008) A clinical study on temporomandibular joint ankylosis in children. J Craniofac Surg 19:1263-1269

7. Guyuron B, Lasa CI Jr (1992) Unpredictable growth pattern of costochondral graft. Plast Reconstr Surg 90:880-886

8. Sawhney CP (1986) Bony ankylosis of the temporomandibular joint: followup of 70 patients treated with arthroplasty and acrylic spacer interposition. Plast Reconstr Surg 77:29

9. Figueroa AA, Gans BJ, Pruzansky S (1984) Long-term follow-up of a mandibular costochondral graft. Oral Surg Oral Med Oral Pathol 58:257-268

10. Ko EW-C, Huang C-S, Chen Y-R (1999) Temporomandibular joint reconstruction in children using costochondral grafts. J Oral Maxillofac Surg 57:789-798

11. Svensson B, Adell R (1998) Costochondral grafts to replace mandibular condyles in juvenile chronic arthritis patients: long-term effects on facial growth. J Cranio-Maxillofac Surg 26:275-285

12. Ross AB (1999) Costochondral grafts replacing the mandibular condyle. Cleft Palate Craniofac J 36:334-339

13. Erdem E, Alkan A (2001) The use of acrylic marbles for interposition arthroplasty in the treatment of temporomandibular joint ankylosis: followup of 47 cases. Int J Oral Maxillofac Surg 30:32-36

14. Mendes D, Jacobs S (1994) Traumatic deformities and reconstruction of the temporomandibular joint. Mastery Plast Reconstr Surg 2:1220-1229

15. Roychoudhury A, Parkash H, Trikha A (1999) Functional restoration by gap arthroplasty in temporomandibular joint ankylosis: a report of 50 cases. Oral Surg Oral Med Oral Pathol Oral Radiol Endod 87:166-169

16. Vieira ACF, Rabelo LRS (2009) Anquilose da ATM em crianças: aspectos de interesse cirúrgico. Rev Cir Traumatol Buco-Maxilo-Fac 9:15-24

17. do Egito Vasconcelos BC, Porto GG, Bessa-Nogueira RV, Do Nascimento M, Marques M (2009) Surgical treatment of temporomandibular joint ankylosis: follow-up of 15 cases and literature review. Med Oral Patol Oral Cir Bucal 14: E34-E38

18. Chossegros C, Guyot L, Cheynet F, Blanc J, Gola R, Bourezak Z et al (1997) Comparison of different materials for interposition arthroplasty in treatment of temporomandibular joint ankylosis surgery: long-term follow-up in 25 cases. Br J Oral Maxillofac Surg 35:157-160

19. Huang I-Y, Lai S-T, Shen Y-H, Worthington P (2007) Interpositional arthroplasty using autogenous costal cartilage graft for temporomandibular joint ankylosis in adults. Int J Oral Maxillofac Surg 36:909-915

20. Danda AK, Ramkumar S, Chinnaswami R (2009) Comparison of gap arthroplasty with and without a temporalis muscle flap for the treatment of ankylosis. J Oral Maxillofac Surg 67:1425-1431

21. Risdon F (1934) Ankylosis of the temporomaxillary joint. J Am Dent Assoc (1922) 21:1933-1937

22. Mercuri LG (2006) Total joint reconstruction-autologous or alloplastic. Oral Maxillofac Surg Clin North Am 18:399-410

23. Petty W (1991) Total joint replacement. J Pediatr Orthoped 12(4):550

24. Driemel O, Braun S, Müller-Richter U, Behr M, Reichert T, Kunkel M et al (2009) Historical development of alloplastic temporomandibular joint replacement after 1945 and state of the art. Int J Oral Maxillofac Surg 38:909-920

\section{Submit your manuscript to a SpringerOpen ${ }^{\circ}$ journal and benefit from:}

- Convenient online submission

- Rigorous peer review

- Immediate publication on acceptance

- Open access: articles freely available online

- High visibility within the field

- Retaining the copyright to your article

Submit your next manuscript at $\gg$ springeropen.com 